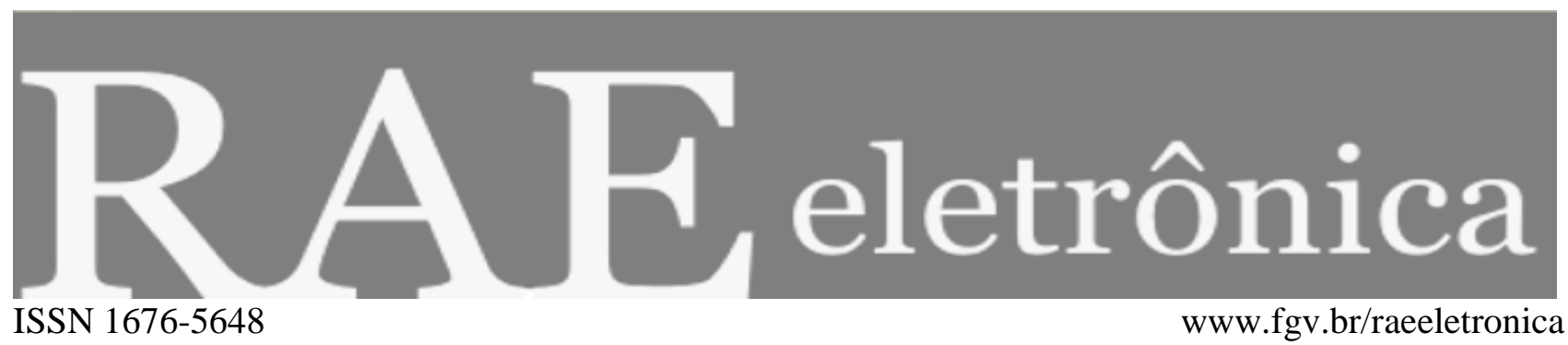

\title{
FÓRUM
}

\section{GESTÃo DE OPERAÇõES NO BRASIL: UMA ANÁLISE DO CAMPO CIENTÍFICO A PARTIR DA REDE SOCIAL DE PESQUISADORES}

\section{OPERATIONS MANAGEMENT IN BRAZIL: AN ANALYSIS OF THE SCIENTIFIC FIELD FROM THE SOCIAL NETWORK OF RESEARCHERS}

\section{GESTIÓN DE OPERACIONES EN BRASIL: UN ANÁLISIS DEL CAMPO CIENTÍFICO A PARTIR} DE LA RED SOCIAL DE INVESTIGADORES

\section{Guilherme Silveira Martins}

Doutorando em Administração de Empresas pela Escola de Administração de Empresas de São Paulo, Fundação Getulio Vargas - São Paulo - SP, Brasil

mr.gsmartins@gmail.com

\section{Luciano Rossoni}

Professor do Programa de Mestrado e Doutorado, Universidade Positivo

lrossoni@gmail.com

\section{João Mário Csillag}

Professor da Escola de Administração de Empresas de São Paulo, Fundação Getulio Vargas - São Paulo SP, Brasil joao.mario.csillag@fgv.br

\section{Michele Esteves Martins}

Mestre em Administração de Empresas pela Escola de Administração de Empresas de São Paulo, Fundação Getulio Vargas - São Paulo - SP, Brasil

micheleemartins@gmail.com

\section{Susana Carla Farias Pereira}

Professora da Escola de Administração de Empresas de São Paulo, Fundação Getulio Vargas - São Paulo SP, Brasil susana.pereira@fgv.br

Recebido em 18.12.2009. Aprovado em 27.07.2010. Disponibilizado em 23.08.2010

Avaliado pelo sistema double blind review

Editor Científico: Organizadores do Fórum de Operações

RAE-eletrônica, v. 9, n. 2, Art. 8, jul/dez. 2010.

http://www.rae.com.br/eletronica/index.cfm?FuseAction=Artigo $\& I D=5612 \&$ Secao=FORUM $\&$ Volume $=9 \& N u m e r o=2 \& A n$ $\mathrm{o}=2010$

(C) Copyright 2010 FGV-EAESP/RAE-eletrônica. Todos os direitos reservados. Permitida a citação parcial, desde que identificada a fonte. Proibida a reprodução total. Em caso de dúvidas, consulte a Redação: raeredacao@ fgv.br; 55 (11) 3799-7898.

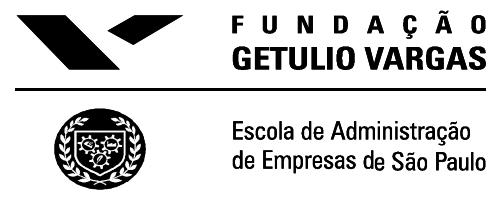




\title{
RESUMO
}

Este estudo analisa a rede de pesquisadores que publicam no campo de Gestão de Operações no Brasil, baseando-se em 2.668 artigos publicados entre 1997 a 2009. As hipóteses do estudo são referenciadas na literatura de redes sociais e de avaliação de campos científicos. Os resultados apontam que, apesar da existência de uma rede fragmentada e pouco densa, há grupos coesos e próximos que dão estabilidade ao campo (redes small worlds). Verificou-se que o número de laços do pesquisador (centralidade de grau) está positivamente associado ao número de artigos publicados, embora afete negativamente sua produtividade. Quando consideramos os laços não redundantes (structural holes), os resultados se mostraram positivos e significativos tanto para a produção quanto para a produtividade. Ademais, observou-se ainda que a centralidade do pesquisador modera o efeito dos laços não redundantes, sendo seu efeito sobre a produtividade maior entre pesquisadores com maior grau de centralidade.

PALAVRAS-CHAVE Gestão de operações, análise de redes sociais, avaliação de campos científicos redes de colaboração científica, produção científica em gestão de operações.

\begin{abstract}
This study examines the network of researchers who publish in the field of Operations Management in Brazil, based on 2668 articles published between 1997 and 2009. The study hypotheses take as reference the literature on social networks and the evaluation of scientific fields. The results indicate that despite the existence of a fragmented and somewhat sparse network there are cohesive and close groups that provide the field with a degree of stability (small world networks). It was found that the number of ties of researchers (degree centrality) is positively associated with the number of articles published, although it negatively affects their productivity. When we consider non-redundant ties (structural holes), the results proved to be positive and significant, both as far as concerning production and productivity. Furthermore, it was also seen that the centrality of the researcher moderates the effect of non-redundant ties, with its effect on productivity being greater among researchers with a greater degree of centrality.
\end{abstract}

KEYWORDS Operations management; social network analysis; evaluation of the scientific field; scientific collaboration networks; scientific production in operations management.

RESUMEN Este estudio analiza la red de investigadores que publican en el campo de Gestión de Operaciones en Brasil, basándose en 2.668 artículos publicados entre 1997 y 2009. Las hipótesis del estudio son referenciadas en la literatura de redes sociales y de evaluación de campos científicos. Los resultados apuntan que, a pesar de la existencia de una red fragmentada y poco densa, hay grupos congruentes y próximos que dan estabilidad al campo (redes small worlds). Se verificó que el número de lazos del investigador (centralidad de grado) está positivamente asociado al número de artículos publicados, aunque afecte negativamente su productividad. Cuando consideramos los lazos no redundantes (structural holes), los resultados se mostraron positivos y significativos tanto para la producción como para la productividad. Además, se observó que la centralidad del investigador modera el efecto de los lazos no redundantes, siendo su efecto sobre la productividad mayor entre investigadores con mayor grado de centralidad.

PALABRAS CLAVE Gestión de operaciones, análisis de redes sociales, evaluación de campo científico, redes de colaboración científica, producción científica en gestión de operaciones. 


\section{INTRODUÇÃO}

O campo de Gestão de Operações experimentou mudanças substanciais desde o estabelecimento das manufaturas no século XIX, com ocasionais crises de identidades (SPRAGUE, 2007). Da Gestão da Fábrica, passando pela Gestão da Produção e chegando a Gestão de Operações, as mudanças se intensificaram, especialmente nas últimas décadas, com grande impacto tanto na atividade empresarial quanto nas atividades de ensino e pesquisa (GUPTA, VERMA, VICTORINO, 2006).

Ao longo dessa evolução, a Gestão de Operações estabeleceu-se como uma das disciplinaschave na maioria das escolas de negócio em todo o mundo (SOTERIOU e outros, 1999), o que motivou os pesquisadores a identificar e estabelecer uma agenda de pesquisa para o campo, com indicações de temas emergentes e de metodologias de investigação (BUFFA, 1980; AMOAKO-GYAMPAH; MEREDITH, 1989; SWAMIDASS, 1996; PANNIRSELVAM e outros, 1999). Outros pesquisadores se preocuparam em realizar uma revisão da produção do campo, em termos de tópicos e métodos empregados para investigar os problemas de pesquisa identificados na área (PRASAD; BABBAR, 2000; ARKADER, 2003; BOYER e outros, 2005; GUPTA e outros, 2006).

Em geral, a avaliação de campos científicos tem como unidade de análise os artigos publicados. Contudo, se olharmos sob a perspectiva de que o conhecimento científico, materializado na publicação desses artigos, é construído socialmente (MERTON, 1973; KUHN, 1978), a partir da cooperação entre pesquisadores no processo de investigação e teorização, o estudo do relacionamento entre eles pode trazer contribuições significativas para o entendimento da dinâmica do campo.

Dessa forma, este artigo se propõe a analisar a estrutura da rede de relacionamento entre os pesquisadores que publicaram nos principais eventos e periódicos nacionais do campo de Gestão Operações no período de 1997 a 2009. A partir dos dados de coautoria e sob a óptica de redes sociais, foram construídas e testadas hipóteses acerca da existência de redes do tipo mundos pequenos (small worlds) e acerca da relação do posicionamento do pesquisador na rede (centralidade e lacunas estruturais, ou structural holes) com o volume de artigos publicados e com a produtividade.

O artigo está estruturado em cinco seções, a contar com esta, introdutória. Na segunda seção são apresentados elementos da análise de redes sociais e constitui-se o referencial para a construção das hipóteses de trabalho. A terceira seção traz as definições metodológicas da pesquisa. Em seguida são apresentados e discutidos os resultados da análise da rede de pesquisadores do Campo de Operações. $\mathrm{Na}$ quinta e última seção encontram-se as conclusões da pesquisa, bem como sugestões para futuros trabalhos. 


\section{COLABORAÇÃo CIENTÍFICA E ANÁLISE DE REDES SOCIAIS}

O potencial da análise de redes sociais na investigação da colaboração científica vem sendo explorado por estudos recentes (NEWMAN, 2001; MOODY, 2004; ROSSONI e MACHADO-DA-SILVA, 2008). Esses trabalhos evidenciaram o crescimento da cooperação entre os pesquisadores, cujas relações não só aumentaram em frequência, mas também em número de colaboradores, possibilitando a formação de redes. Assim, a avaliação da construção do conhecimento científico, que tradicionalmente era baseada no trabalho individual, pode partir de uma nova perspectiva: o conjunto de relacionamentos entre pesquisadores. É comum que estudos deste tipo considerem como base analítica a cooperação entre cientistas em termos de coautoria - i.e. participação de dois ou mais autores na produção de um estudo - por se configurar como uma manifestação formal de colaboração intelectual na pesquisa científica (GLANZEL e SCHUBERT, 2004).

A primeira hipótese deste estudo parte da lógica de grupos de pesquisadores coesos em uma rede de colaboração científica. Dentre as diversas configurações estruturais de redes sociais, uma em especial vem chamando a atenção na avaliação de campos científicos (MOODY, 2004): redes mundos pequenos (small worlds). Ao contrário de considerar que o processo de desenvolvimento científico ocorre segundo uma lógica de fragmentação, sem interface entre os diferentes grupos, esta considera que há ligações entre eles que mantêm um nível de coesão para que as atividades se tornem familiares entre os membros dos diferentes grupos (UZZI e SPIRO, 2005). Subconjuntos coesos de atores apresentam laços relativamente fortes, diretos, intensos e frequentes (WASSERMAN e FAUST, 1994), o que lhes permite possuir normas, valores, orientações e subculturas próprias (SCOTT, 2000).

As propriedades de small worlds propiciam elementos para a análise da durabilidade das estruturas de relacionamento, bem como de práticas e valores científicos (KOGUT e WALKER, 2001; ROSSONI e MACHADO-DA-SILVA, 2008), o que é importante para melhor entendimento da relação entre estruturas locais e globais da rede. Estruturas de rede do tipo small world foram verificadas nas áreas de Sociologia por Moody (2004) e na de Física por Newman (2001). Em Administração, no contexto brasileiro, Rossoni e Machado-da-Silva (2008) encontraram resultados semelhantes para a área de Estudos Organizacionais e Estratégia.

A origem da área de Gestão de Operações também suporta a construção da primeira hipótese. Conforme Sprague (2007), as primeiras escolas de Administração foram fundadas ainda no século XIX, 
tanto nos Estados Unidos quanto na Europa, com foco na Gestão da Produção, e contaram com professores da área de Engenharia. Tal interface ainda é grande, o que confere à área uma diversidade de abordagens temáticas e metodológicas na geração de conhecimento. Assim, tal diversidade tende a levar a uma rede de pesquisadores fragmentada e pouco densa ao nível global, mas coesa ao nível local, por compartilhamento de abordagens na condução do trabalho científico.

Com base nos argumentos teóricos e nas evidências empíricas de outros campos científicos apresentados, a seguinte hipótese de pesquisa foi verificada:

$\mathrm{H}_{1}$ : A rede de cooperação do campo de Operações no Brasil apresenta estruturas do tipo small worlds.

A inserção de indivíduos em uma grande rede ou o estabelecimento de novos laços nessa rede geralmente não ocorre de maneira aleatória (BARABÁSI e ALBERT, 1999). A probabilidade de um novo ator social se conectar àqueles já participantes dos relacionamentos não apresenta distribuição uniforme, mas amplia-se de acordo com o aumento do número de laços que um determinado ator possui. Quanto mais ligações um ator possui com outros, mais centralmente ele se posiciona na rede e, por essa razão, maiores serão suas chances de cultivar novos relacionamentos (LEE e BOZEMAN, 2005).

A centralidade de um ator é frequentemente utilizada para analisar sua importância na rede. Dentre várias medidas, optou-se por trabalhar com a centralidade de grau (degree), pois esta permite uma avaliação da atividade local dos atores na rede e aponta, objetivamente, o número de pesquisadores que cada autor publicou em conjunto. Formalmente, a centralidade de grau é definida pelo número de laços adjacentes de um ator em uma rede (WASSERMAN e FAUST, 1994). Nesse caso, quanto maior o grau de centralidade, maiores a possibilidade de um pesquisador produzir artigos, já que conta com outros para dividir e compartilhar a atividade de investigação e produção. Assim, foi construída a segunda hipótese:

$\mathrm{H}_{2 \mathrm{a}}$ : Há relação positiva e significativa entre o número de laços do pesquisador e o número de artigos que ele publicou. 
O número de artigos publicados, apesar de mostrar o empenho de determinado pesquisador em contribuir para a área, pode ser questionado quando se busca entender sua produtividade. Braun, Glänzel e Schubert (2001) e Rossoni e Machado-da-Silva (2008) relacionaram a produção dos pesquisadores ao número de coautores com quem colaboraram e demonstraram que, mesmo havendo um aumento acentuado no número absoluto de artigos publicados por autor nos últimos anos, quando se ponderou o total de artigos publicados na área pelo total de pesquisadores, verificou-se que a produtividade deles vem caindo. Todavia, apesar da queda de produtividade no nível geral, há evidências de que, em algumas áreas, o aumento na colaboração está associado ao aumento na produtividade (LEE e BOZEMAN, 2005), provavelmente porque os pesquisadores mais centrais tendem a ser mais experientes no processo de produção do conhecimento, o que pode indicar maior competência em gerenciar seus recursos relacionais. Em face do exposto, se os pesquisadores individualmente se beneficiam da colaboração para ter maior produtividade (LEE e BOZEMAN, 2005), espera-se que aqueles mais centrais apresentem maior probabilidade de ter maior produtividade, equacionada pelo número de trabalhos publicados em relação ao total de colaboradores. Delineia-se daí a seguinte hipótese:

$\mathrm{H}_{2 b}$ : Há relação positiva e significativa entre o número de laços do pesquisador e sua produtividade.

Além da quantidade de laços diretos desenvolvidos por cada autor (i.e. centralidade de grau), é possível avaliar a qualidade desses laços por meio da sua eficiência. Entende-se por laços eficientes aqueles não redundantes entre os contatos diretos de um ator. Pressupõe-se que, quanto maior a diversidade dos laços de um indivíduo na rede, maior o acesso a diferentes recursos do grupo social que interage com maior frequência (LIN, 2001; MIZRUCHI, 1996). Para Burt (1992), os relacionamentos não redundantes entre dois contatos caracterizam structural holes (lacunas estruturais). Quanto menor o número de laços redundantes, maior o número de lacunas estruturais na rede, havendo menor redundância de informação. O elemento fundamental das lacunas estruturais está na extensão em que a estrutura social de uma arena competitiva cria oportunidades para certos agentes, por meio de seus relacionamentos. Nesses termos, o controle e a participação na difusão da informação definem o capital social das lacunas estruturais (BURT, 2000). 
Se laços não redundantes (i.e. eficientes) permitem ao pesquisador participar de diferentes grupos e possibilitam o acesso a diferentes recursos, espera-se que esses laços potencializem sua produção de trabalhos científicos. Por um lado, isso significa que as lacunas estruturais aumentam as suas chances de produzir maior número de artigos em coautoria com outros pesquisadores com habilidades e recursos complementares ao seu. Por outro, isso indica que, se há chances de o conhecimento e os recursos compartilhados a partir dos laços não redundantes serem uma fonte de vantagens para o pesquisador com maior proporção desses laços, espera-se que os indivíduos que consigam desenvolver número maior de laços não redundantes sejam mais produtivos que os demais. Sendo assim, temos as seguintes hipóteses:

$\mathrm{H}_{3 \mathrm{a}}$ : Existe relação positiva e significativa entre a eficiência dos laços do pesquisador e o número de artigos que ele publicou.

$\mathrm{H}_{3 \mathrm{~b}}$ : Existe relação positiva e significativa entre a eficiência dos laços do pesquisador e sua produtividade.

Ainda assim, uma situação fica passível de questionamento: pesquisadores que possuem um pequeno número de laços (i.e. menor centralidade de grau) recebem os mesmos benefícios dos laços não redundantes que indivíduos com número elevado de laços? Espera-se que a resposta seja não. $\mathrm{O}$ primeiro argumento é lógico: se um indivíduo tem somente um único colaborador, não há como ele ter laço redundante com ninguém, portanto a totalidade de seus laços é não redundante. O segundo argumento é relacional: espera-se que as trocas relacionais em termos de coautoria sejam desiguais, e que indivíduos com maior destaque no campo (no nosso caso, com maior número de colaboradores) se beneficiem em maior grau dos relacionamentos do que seus parceiros menos centrais (ver, por exemplo, o efeito de obliteração na ciência apontado por Merton, 1968, 1988). Por fim, o terceiro argumento envolve as capacidades dos indivíduos enquanto agentes aptos a aproveitar as vantagens que as lacunas estruturais proporcionam. Indivíduos com maior número de colaboradores tendem a ser mais experientes que indivíduos com poucos laços, já que um maior número de colaboradores está atrelado à maior habilidade em conduzir o processo de interação necessário para a produção do conhecimento.

Em face do exposto, espera-se que os indivíduos que tenham maior centralidade em termos de número de colaboradores aproveitem melhor os benefícios das lacunas estruturais, o que lhes 
proporciona um aumento do número de artigos publicados e de sua produtividade. Portanto, as últimas hipóteses deste estudo são:

$\mathrm{H}_{4 \mathrm{a}}$ : $\mathrm{O}$ efeito da eficiência dos laços do pesquisador sobre o número de artigos publicados é moderado pela centralidade de grau, de forma que o efeito da eficiência dos laços é maior entre os pesquisadores com alta centralidade do que entre os pesquisadores com baixa centralidade.

$\mathrm{H}_{4 \mathrm{~b}}$ : $\mathrm{O}$ efeito da eficiência dos laços do pesquisador sobre a produtividade é moderado pela centralidade de grau, de forma que o efeito da eficiência dos laços é maior entre os pesquisadores com alta centralidade do que entre os pesquisadores com baixa centralidade.

\section{PROCEDIMENTOS METODOLÓGICOS}

Para alcançar os objetivos declarados neste trabalho, foi desenvolvido um estudo de natureza descritiva e explicativa. Ele assume um caráter descritivo ao procurar caracterizar as estruturas de relacionamento entre pesquisadores do campo de Operações no Brasil e, a partir delas, verificar sua conformidade com configurações do tipo small worlds. E é explicativo, pois, a partir da análise das redes de cooperação, pretende compreender se os pesquisadores com maior centralidade e com maior eficiência dos laços tendem a ter maior produção e produtividade científica que os demais. O nível de análise da pesquisa é a rede formada por pesquisadores das áreas de Gestão de Operações, e a unidade de análise é cada pesquisador individualmente. A abordagem utilizada neste trabalho permite classificá-lo ainda como um metaestudo, já que ele busca caracterizar a atividade de pesquisa do campo de Gestão de Operações a partir dos artigos produzidos pelos pesquisadores da área, o que possibilitou o delineamento de redes de coautoria. Justifica-se o uso da coautoria devido à confiabilidade das informações e à disponibilidade de acesso, já que outros tipos de relações entre pesquisadores dificilmente estão organizados de forma sistemática (ACEDO e outros, 2006).

\section{Dados e amostra}


Como estratégia de coleta de dados, foi desenvolvida uma pesquisa do tipo documental em artigos científicos publicados nos principais eventos e periódicos brasileiros no período de 1997 a 2009 , conforme a Tabela 1.

Tabela 1 - Fontes da pesquisa documental

\begin{tabular}{|c|c|c|c|}
\hline FONTE & $\begin{array}{c}\text { EDIÇÃO / } \\
\text { ORGANIZAÇÃO }\end{array}$ & $\begin{array}{l}\text { PERÍODO } \\
\text { DE ANÁLISE }\end{array}$ & $\begin{array}{l}\text { NÚMERO DE } \\
\text { ARTIGOS }\end{array}$ \\
\hline $\begin{array}{l}\text { EnAnpad (área GOL - Gestão de } \\
\text { Operações e Logística) }{ }^{1}\end{array}$ & ANPAD & 1997 a 2009 & 386 \\
\hline SIMPOI $^{1}$ & FGV-EAESP & 1998 a $2009^{3}$ & 1.724 \\
\hline $\begin{array}{l}\text { RAE- revista de administração de } \\
\text { empresas }{ }^{2}\end{array}$ & FGV-EAESP & 1997 a 2009 & 30 \\
\hline RAE-eletrônica ${ }^{2}$ & FGV-EAESP & 2002 a $2009^{3}$ & 12 \\
\hline $\begin{array}{l}\text { Revista de Administração } \\
\text { Contemporânea }(R A C)^{2}\end{array}$ & ANPAD & 1997 a 2009 & 31 \\
\hline RAC Eletrônica ${ }^{2}$ & ANPAD & 2007 a $2009^{3}$ & 6 \\
\hline RAUSP ${ }^{2}$ & FEA/USP & 1997 a 2009 & 50 \\
\hline RAUSP Eletrônica ${ }^{2}$ & FEA/USP & 2008 e $2009^{3}$ & 0 \\
\hline Brazilian Administration Review (BAR) ${ }^{2}$ & ANPAD & 2004 a $2009^{3}$ & 10 \\
\hline Revista Gestão e Produção ${ }^{1}$ & UFSCar $^{4}$ & 1997 a 2009 & 397 \\
\hline $\begin{array}{l}\text { Journal of Operations and Supply Chain } \\
\text { Management (JOSCM })^{1}\end{array}$ & FGV-EAESP & 2008 e $2009^{3}$ & 22 \\
\hline TOTAL & & 1997 a 2009 & 2.668 \\
\hline
\end{tabular}

Notas: (1) seleção de todos os artigos publicados; (2) foram selecionados apenas os artigos cujo escopo temático pertencia ao campo de Gestão de Operações; (3) primeira edição posterior a 1997; (4) embora editada por uma escola de Engenharia, optou-se por incluir a revista na análise da rede de Operações pelo fato de os pesquisadores participarem dos fóruns de Gestão - os dados apontam que $77 \%$ dos autores que publicaram nesse periódico também publicaram em outro evento ou periódico pesquisado.

Das publicações, foram extraídos os dados do pesquisador que, sozinho ou em conjunto com outros autores, publicou algum artigo científico no período analisado. Os dados foram tabulados no Microsoft Excel $2007^{\circledR}$, o que propiciou a construção das matrizes quadradas que refletem a estrutura das redes entre os pesquisadores, cuja análise foi conduzida com o apoio dos softwares Ucinet 6 e Pajek 1.24.

\section{Variáveis}


Autorias. Esta foi utilizada como uma das variáveis dependentes deste trabalho, a exemplo de outros estudos (LEE e BOZEMAN, 2005; ROSSONI e GUARIDO-FILHO, 2007, 2009; ROSSONI e HOCAYEN-DA-SILVA, 2008). O número de autorias de um pesquisador é o número de artigos que ele publicou em determinado período e em determinada área.

Produtividade acadêmica. Para avaliar a segunda variável dependente, optou-se por utilizar os critérios de Braun, Glänzel e Schubert (2001) e Lee e Bozeman (2005), em que a produtividade de um pesquisador é dada pelo número de artigos publicados por ele em determinado período, dividido pelo total de colaboradores, somando-se, também, o próprio autor. Formalmente, tem-se então: artigos/(coautores +1$)$.

Centralidade de grau (degree). Constitui-se como uma das variáveis independentes deste estudo e é definida como o número de laços $n$ que um determinado ator $i$ possui (WASSERMAN e FAUST, 1994). Os laços de um pesquisador são todos os indivíduos que publicaram um ou mais artigos conjuntamente com ele. Para tanto, calculamos a centralidade de grau por meio da matriz de relacionamentos de forma simétrica e binária.

Eficiência dos laços (\% structural holes). Para avaliar o efeito dos laços fracos, foi utilizada a medida de eficiência dos laços (BURT, 1992), que mensura o número de laços não redundantes (EffSize) em relação ao total de contatos $n$ de um ator $i$. Com dados binários, é possível utilizar a forma simplificada da equação desenvolvida por Borgatti (1997). Formalmente, considerando que um ator $i$ apresenta $n$ contatos, é possível avaliar o número de laços redundantes por meio da equação $D_{\text {alters }}=2 l / n$, em que $l$ é o número de laços entre $n$ (alters). Já que $D_{\text {alters }}$ indica o total de laços redundantes, considera-se como laços não redundantes EffSize como $n-D_{\text {alters. Dessa forma, a }}$ proporção de laços não redundantes (efficiency) é dada por EffSize/n. Essa medida foi utilizada por não apresentar alta correlação com o número de contatos e por sua representatividade em relação ao engajamento nos laços fracos (GRANOVETTER, 1973).

\section{MÉTODOS DE ANÁLISE}

A análise dos dados teve uma abordagem quantitativa, apoiada tanto na análise de redes sociais quanto em análises estatísticas, a partir do uso de informações sobre o relacionamento entre unidades analíticas. Dessa forma, foi possível avaliar tanto os aspectos descritivos dos relacionamentos de coautoria quanto 
realizar procedimentos estatísticos complementares na investigação de associações entre as variáveis estudadas (WASSERMAN e FAUST, 1994; SCOTT, 2000), sendo estes expostos mais detalhadamente a seguir.

Análise das redes small worlds. Para avaliar as propriedades estruturais da rede de relações entre pesquisadores, tomaram-se como base os parâmetros estabelecidos por Watts e Strogatz (1998) e Watts (1999). Segundo os autores, as redes small worlds apresentam distância média entre os atores menor do que em redes aleatórias, ao mesmo tempo que o coeficiente de agrupamento deve ser maior. Para que uma rede seja considerada um mundo pequeno é necessário se ater a duas propriedades da rede: distância média $(L-$ length $)$ e coeficiente de agrupamento $(C C-$ clustering coefficient $)$. A distância média $(L)$ é a extensão do menor caminho que liga um ator a todos os outros da rede, calculada para o conjunto de pares de atores. Altos valores de $L$ indicam que os recursos, tais como informações, devem passar por um grande número de intermediários para viajar entre atores na rede.

O coeficiente de agrupamento $(C C)$ consiste no número de ligações entre os vizinhos imediatos de um pesquisador, em comparação ao número máximo de laços que possam ter. Formalmente, podemos definir o coeficiente de agrupamento $C C$ como $3 x$ número de cliques/número de trios conectados. Ele pode variar de zero, redes locais totalmente não agrupadas, a 1, redes totalmente agrupadas. Altos valores de $C C$ indicam que a rede é composta por grupos locais densamente interligados.

Verifica-se empiricamente se uma rede é um mundo pequeno a partir da comparação dos valores destes dois parâmetros $(L$ e $C$ C) para a rede real de Operações e para uma rede aleatória com o mesmo número de pesquisadores. No caso das redes aleatórias, $L_{e s p}=\ln (n) / \ln (k)$ e $C C_{e s p}=k / n$, onde $L_{e s p}$ é a distância média esperada, $n$ é o número de nós (pesquisadores) na rede e $k$ é a média de laços dos atores da rede (WATTS, 1999). Para que uma rede tenha configuração do tipo small world, a distância média real ou observada é similar à calculada na rede aleatória $\left(L_{o b s .} \sim L_{e s p}\right)$, enquanto o valor do coeficiente de agrupamento da rede real é muito maior que o mesmo valor para a rede aleatória $\left(C C_{o b s .}>>C C_{e s p}\right.$.). Com base em Kogut e Walker (2001) e Davis e outros (2003), foi utilizado o seguinte resumo estatístico para indicar a presença ou ausência de small world: $S W Q=\left(C C_{\text {obs. }} / C C_{\text {esp. }}\right) /$ $\left(L_{o b s} / L_{e s p .}\right)$. A rede é considerada um small world quando $S W Q$ é muito maior que um $(S W Q>>1)$ (DAVIS e outros, 2003). Seguindo as orientações de Moody (2004) e Newman (2001), considerou-se somente o componente principal para o cálculo de small world. Componentes são sub-redes em que os 
nós estão conectados entre si; o componente principal é a sub-rede com maior número de atores (WASSERMAN e FAUST, 1994).

Análise de regressão. Buscou-se avaliar o quanto a centralidade de grau do pesquisador explica em termos de variância o número de autorias (hipótese $2_{\mathrm{a}}$ ) e a produtividade do pesquisador (hipótese $2{ }_{b}$ ) por meio de regressão linear múltipla (método $O L S$ ), operacionalizada por meio do software SPSS $17^{\circledR}$. Foram aceitos como significativos os resultados com nível de confiança de $95 \%$ ( $<<0,05$ ). Procedimento semelhante foi adotado para o estudo da terceira hipótese: avaliou-se o coeficiente de explicação da eficiência dos laços sobre o número de artigos publicados (hipótese $3_{\mathrm{a}}$ ) e sobre a produtividade (hipótese $3 \mathrm{~b}$ ). Posteriormente, as análises da influência da eficiência dos laços foi repetida considerando dois grupos distintos: um com autores com número grande de colaboradores (alto degree), outro com número menor de colaboradores (baixo degree). Esse procedimento foi necessário para se avaliar o efeito moderador da colaboração.

Análise do efeito moderador. Para testar as hipóteses de número quatro, avaliou-se o efeito moderador da cooperação sobre a relação entre eficiência dos laços e variáveis dependentes. Uma variável é tida como moderadora quando a força do relacionamento entre duas variáveis depende dela (PREACHER, RUCKER, HAYES, 2007). Baron e Kenny (1986) sugerem que o efeito moderador pode ser avaliado por meio da interação entre a variável independente e a moderadora. No entanto, como aponta Arnold (1982), esse procedimento pode gerar interpretações equivocadas porque, diante da colinearidade entre o termo de interação com as variáveis moderadora e independente, os coeficientes ficam enviesados. Para resolver o problema, o autor sugere dividir a amostras em dois subgrupos: um com os casos que apresentam valores altos ou a presença do elemento moderador e outro com valores baixos ou a ausência do elemento moderador. A partir das análises de regressões, os coeficientes das variáveis independentes devem ser comparados entre si por meio do teste do qui quadrado, pela fórmula:

$$
\operatorname{chi}{ }^{2}{ }_{m p}=\frac{\left(\operatorname{bet} \alpha_{2}-b e t \alpha_{1}\right)^{2}}{\left[\left(s e_{2} \mathbf{I}^{2}+5 \varepsilon_{1}^{2}\right)\right.}
$$

O valor do beta $_{1}$ é o coeficiente da variável independente no grupo 1; o do beta $a_{2}$, o coeficiente da variável independente no grupo $2 ; s e_{1}$, o erro padrão do coeficiente do grupo $1 ; s e_{2}$, o erro padrão do coeficiente do grupo 2. Com o valor do qui quadrado calculado para cada uma das comparações dos coeficientes entre os dois grupos, aceita-se o efeito moderador quando ele é significativo. Assim, a 
amostra foi dividida em dois grupos: um que contém pesquisadores com vários colaboradores (média de 10,6 laços de coautoria, totalizando 164 pesquisadores), denominado "alto degree"; e outro, com pesquisadores com número menor de colaboradores (média de 2,07 laços de coautoria, englobando 2.984 pesquisadores), "baixo degree". Para dividir esses grupos em duas porções, utilizamos o procedimento de análises de clusters por meio do método k-means, objetivando maximizar a homogeneidade dentro dos grupos. Destaca-se que tal procedimento é adequado quando temos o número de grupos a priori.

\section{CONFIGURAÇÃO DA REDE DE PESQUISADORES DO CAMPO DE OPERAÇÕES}

O campo de Gestão de Operações no Brasil experimentou um crescimento constante ao longo do período analisado. Tal fenômeno pode ser constatado visualmente na Figura 1 e nos dados estatísticos da rede presentes na Tabela 2. A Figura 1 ilustra as redes de relações entre os pesquisadores em diferentes períodos. Nela, cada nó representa um pesquisador, cujos laços expressam relações de coautoria.

As redes que retratam os relacionamentos do campo de Operações não são totalmente conectadas e apresentam várias pequenas sub-redes em torno de uma rede maior, chamada de componente principal. Em tese, se o campo científico se apresenta mais aglomerado, com um grande número de pesquisadores em um mesmo componente, maior é o potencial de troca de informações entre eles. Nessas circunstâncias, pode ocorrer maior similaridade de práticas de pesquisa e de definição de temáticas, o que supostamente provoca maior homogeneidade no campo. Ao contrário, a existência de vários grupos de pesquisadores pode indicar fragmentação do campo (MOODY, 2004).

O período três (2006-2009) é responsável por quase metade do volume de artigos publicados em todo o período (Tabela 2). Observa-se ainda que o número de pesquisadores mais que dobrou no período três em relação ao primeiro. Com o campo mais populoso, aumenta-se a possibilidade de os pesquisadores efetuarem laços de coautorias, o que pode ser observado no discreto aumento na média de laços dos pesquisadores. Para todo o período, cada autor colaborou em média com 2,5 pesquisadores para a produção de artigos. 
Figura 1 - Desenvolvimento da rede de pesquisadores do campo de operações
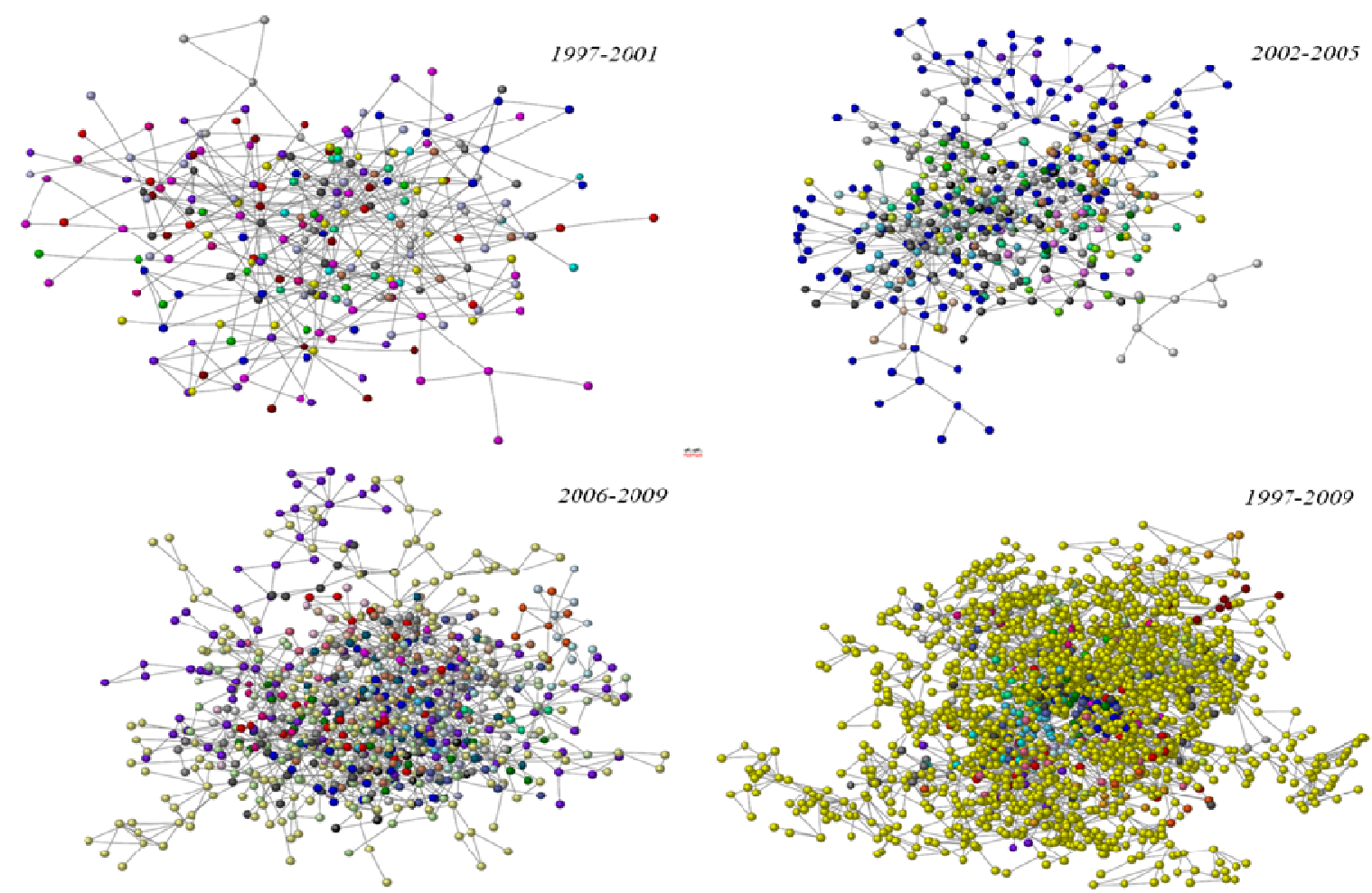

Notas: Os nós representam pesquisadores, e os laços são formados a partir de coautorias. As cores dos nós indicam diferentes componentes.

Tabela 2 - Estatística descritiva das estruturas de relações do campo de operações

\begin{tabular}{|l|c|c|c|c|}
\hline \multirow{2}{*}{} & \multicolumn{1}{c}{ PERÍODO 1 } & \multicolumn{1}{c}{ PERÍODO 2 } & \multicolumn{1}{c}{ PERÍODO 3 } & \multicolumn{1}{c}{ TOTAL } \\
\cline { 2 - 5 } \multicolumn{1}{c|}{} & $\mathbf{1 9 9 7 - 2 0 0 1}$ & $\mathbf{2 0 0 2 - 2 0 0 5}$ & $\mathbf{2 0 0 6 - 2 0 0 9}$ & $\mathbf{1 9 9 7 - 2 0 0 9}$ \\
\hline Total de artigos & 557 & 904 & 1.207 & 2.668 \\
\hline Total de pesquisadores & 766 & 1.245 & 1.801 & 3.148 \\
\hline Média de laços por autor & 1,958 & 2,051 & 2,382 & 2,517 \\
\hline Densidade & $0,26 \%$ & $0,16 \%$ & $0,13 \%$ & $0,08 \%$ \\
\hline Total de componentes & 162 & 232 & 334 & 413 \\
\hline Total Pesquisadores no componente principal & $29(3,8 \%)$ & $130(10,4 \%)$ & $224(12,4 \%)$ & $1.526(48,5 \%)$ \\
\hline Total Pesquisadores isolados & $105(13,7 \%)$ & $84(6,7 \%)$ & $56(3,1 \%)$ & $176(5,6 \%)$ \\
\hline
\end{tabular}

Ainda com base na Tabela 2, observa-se que a proporção de pesquisadores que publicavam isolados caiu e representava $5,6 \%$ do total. Verifica-se ainda que o indicador de densidade dos relacionamentos na rede global foi baixo em todos os períodos analisados. Essa medida indica o percentual dos laços possíveis na rede que são efetivamente realizados. Na rede total (1997-2009), o indicador foi de 0,08\% 
e caiu do primeiro para o último período com o aumento do número de pesquisadores na rede. Tal padrão de densidade é comparável ao de outras redes de pesquisadores de ciências sociais (MOODY, 2004; ACEDO e outros, 2006) e pouco inferior ao encontrado na rede de Organizações e Estratégia no Brasil, identificado por Rossoni e Machado-da-Silva (2008) - 0,11\%, possivelmente pelo maior tamanho da rede na amostra estudada em Operações, pois tamanho da rede e densidade covariam inversamente (WATTS, 1999).

Com o aumento da rede, o número de componentes aumentou consideravelmente, embora em uma escala menor que o número de pesquisadores que atuam no campo. Isso é um indício de que os pesquisadores tendem a entrar no campo por meio de laços de coautoria com pesquisadores já atuantes, possivelmente por meio de relações de orientação de programas de mestrado e doutorado. Ao longo dos períodos de análise, o componente principal da rede se tornou mais representativo. Na rede do período total analisado, o componente principal é composto por 1.526 pesquisadores, o que equivale a 48,5\% dos indivíduos que publicaram no campo. A Tabela 3 apresenta os resultados de estudos em redes de colaboração científica de diferentes campos. Nota-se que os indicadores do campo Gestão de Operações possuem um padrão que o aproxima daquele encontrado em outros campos de Ciências Sociais. Nestes, quando são analisados o tamanho do componente principal e a média de laços por autor, a conectividade entre os pesquisadores se mostra inferior à dos campos de Medicina, Física e Matemática.

Tabela 3 - Comparação da rede de diferentes campos científicos

\begin{tabular}{|c|c|c|c|c|c|c|c|}
\hline & \multicolumn{2}{|c|}{ BRASIL } & \multicolumn{5}{|c|}{ INTERNACIONAL } \\
\hline & $\begin{array}{c}\text { GESTÃO DE } \\
\text { OPERAÇÕES } \\
\end{array}$ & $\begin{array}{c}\text { ESTRATÉGIA } \\
\text { E ESTUDOS } \\
\text { ORGANIZACIONAIS }^{2} \\
\end{array}$ & $\begin{array}{l}\text { ADMINIS- } \\
\text { TRAÇÃO }^{3}\end{array}$ & MEDICINA $^{4}$ & FÍSICA $^{5}$ & $\begin{array}{c}\text { MATEMÁ- } \\
\text { TICA }^{5}\end{array}$ & SOCIOLOGIA $^{6}$ \\
\hline Período & $1997-2009$ & 1997-2005 & $1980-2002$ & $\begin{array}{l}1995- \\
1999\end{array}$ & $\begin{array}{l}1995- \\
1999\end{array}$ & 1995-1999 & 1963-1999 \\
\hline $\begin{array}{l}\text { Total de } \\
\text { pesquisadores }\end{array}$ & 3.148 & 2.072 & 10.176 & 1.520 .251 & 52.909 & 253.339 & 197.976 \\
\hline $\begin{array}{l}\text { Média de laços por } \\
\text { autor }\end{array}$ & 2,52 & 2,25 & 2,86 & 16,93 & 9,27 & 3,90 & 1,88 \\
\hline $\begin{array}{l}\% \text { pesquisadores no } \\
\text { componente principal }\end{array}$ & $48,5 \%$ & $37,9 \%$ & $45,4 \%$ & $92,6 \%$ & $85,4 \%$ & $82,0 \%$ & $34,5 \%$ \\
\hline
\end{tabular}

Fontes: (1) Resultados da pesquisa; (2) Rossoni e Machado-da-Silva (2008); (3) Acedo e outros (2006); (4) Newman (2001); (5) Newman (2004); (6) Moody (2004).

A presença de pesquisadores em um mesmo componente não significa necessariamente que eles se reconheçam, muito menos que formem grupos coesos. Entretanto, somente a partir da identificação do 
componente principal é que se torna possível explorar a primeira hipótese: se o campo de Operações apresenta uma configuração estrutural do tipo small worlds.

A Tabela 4 apresenta os resultados do teste. Observa-se que em todos os períodos a rede apresentou um alto coeficiente de agrupamento $(C C)$, superior ao coeficiente esperado para uma rede aleatória. Para a rede de todo o período, a razão entre os coeficientes observado e esperado supera 300. Isso indica a presença de clusters coesos de pesquisadores. Contudo, quando a análise recai sobre a distância média $(L)$ entre os indivíduos, verifica-se que ela foi maior que a esperada em todos os períodos de análise. Assim, os indivíduos estão mais distantes uns dos outros do que se esperaria encontrar em uma rede aleatória. Mesmo diante desse maior distanciamento, o coeficiente small world $(S W Q)$ se mostrou significativo $(S W Q>>1)$ para todos os períodos analisados, especialmente quando os 13 anos de análise são considerados $(S W Q=209,5)$. Assim, a maior distância entre os pesquisadores foi compensada pela maior coesão dos grupos. Com isso, a hipótese 1 é aceita: a rede de pesquisadores no Campo de Operações no Brasil segue a lógica da dinâmica de small worlds.

A configuração de small worlds foi encontrada por Rossoni e Machado-da-Silva (2008) no campo de Organizações e Estratégia e se opõe aos resultados de Rossoni e Hocayen-da-Silva (2008) no campo de Administração da Tecnologia de Informação, possivelmente por aspectos relacionados à maturidade dos campos. Esse resultado para o campo de Gestão de Operações evidencia empiricamente a cooperação entre os pesquisadores da área em termos de coautoria e difere de outros estudos realizados no Brasil, que afirmaram a quase inexistência de colaboração na área da Administração (BULGACOV e VERDU, 2003; RODRIGUES e CARRIERI, 2001). Embora não seja possível fazer maiores ilações acerca do desenvolvimento da pesquisa, em termos da qualidade dos artigos produzidos no campo, o fato de os pesquisadores formarem uma rede coesa - ainda que localmente, na formação de clusters - pode potencializar a troca de informações, de modo a fomentar o compartilhamento de significados ou o intercâmbio de conceitos e técnicas de pesquisa.

O papel central dos indivíduos na rede é investigado na segunda hipótese do estudo. A centralidade foi discutida em termos do número de laços de primeiro nível estabelecidos por um pesquisador (centralidade de grau - degree). A Tabela 5 apresenta os pesquisadores mais centrais e a eficiência dos laços efetuados por eles. Destaca-se ainda que os laços desenvolvidos por esses pesquisadores possuem alto grau de eficiência; dessa forma, grande parte desses laços são exclusivos desses pesquisadores e, portanto, não redundantes aos laços dos demais pesquisadores de sua rede, o 
FÓRUM - GESTÃO DE OPERAÇÕES NO BRASIL: UMA ANÁLISE DO CAMPO CIENTÍFICO A PARTIR DA REDE SOCIAL DE PESQUISADORES

Guilherme Silveira Martins - Luciano Rossoni - João Mário Csillag - Michele Esteves Martins - Susana Carla Farias Pereira

que lhe confere, em tese, poder de intermediação dos relacionamentos e acesso a diferentes tipos de informação e recurso.

Tabela 4 - Estatística de small worlds

\begin{tabular}{|c|c|c|c|c|c|}
\hline \multirow[t]{2}{*}{ MEDIDAS } & & \begin{tabular}{|c} 
PERÍODO \\
1 \\
\end{tabular} & $\begin{array}{c}\text { PERÍODO } \\
2 \\
\end{array}$ & $\begin{array}{c}\text { PERÍODO } \\
\mathbf{3} \\
\end{array}$ & TOTAL \\
\hline & & 1997-2001 & $2002-2005$ & 2006-2009 & 1997-2009 \\
\hline Densidade & & $10,84 \%$ & $2,41 \%$ & $1,42 \%$ & $0,21 \%$ \\
\hline \# laços & & 88 & 404 & 708 & 4.926 \\
\hline \# pesquisadores & $n$ & 29 & 130 & 224 & 1.526 \\
\hline Média de laços por pesquisador & $k$ & 3,034 & 3,108 & 3,161 & 3,228 \\
\hline Coeficiente de agrupamento observado & $C C_{\text {obs. }}$ & 0,673 & 0,690 & 0,686 & 0,707 \\
\hline Coeficiente de agrupamento esperado & $C C_{\text {esp. }}=k / n$ & 0,105 & 0,024 & 0,014 & 0,002 \\
\hline$C C_{o b s} / C C_{e s p}$ & & $6,4 \checkmark$ & $28,9 \checkmark$ & $48,6 \checkmark$ & $334,2 \checkmark$ \\
\hline Distância média observada & $L_{\text {Obs }}$ & 3,081 & 5,128 & 6,048 & 9,976 \\
\hline Distância média esperada & $L_{e s p .}=(\ln (n) / \ln (k))$ & 3,034 & 4,292 & 4,702 & 6,255 \\
\hline $\mathrm{DM}_{\text {Obs. }} / \mathrm{DM}_{\mathrm{esp}}$ & & $1,016 \times$ & $1,195 \times$ & $1,286 \times$ & $1,595 \times$ \\
\hline SWQ: Coeficiente Small World & 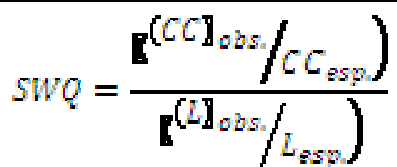 & $6,3 \checkmark$ & $24,2 \checkmark$ & $37,8 \checkmark$ & $209,5 \checkmark$ \\
\hline
\end{tabular}

Notas: O símbolo $(\checkmark)$ indica que a métrica está em acordo com as premissas de small worlds. Já o símbolo $(\mathbf{x})$ indica que a métrica não contribui para a configuração do tipo small world. Os cálculos são válidos para o componente principal, conforme Newman (2001) e Moody (2004).

Tabela 5 - Pesquisadores com maior centralidade de grau, eficiência de seus laços e sua produção (1997-2008)

\begin{tabular}{|l|c|c|c|}
\hline \multicolumn{1}{|c}{ PESQUISADOR } & CENTRALIDADE DE GRAU & $\begin{array}{c}\text { EFICIÊNCIA DOS } \\
\text { LAÇOS }\end{array}$ & $\begin{array}{c}\text { NÚMERO DE ARTIGOS } \\
\text { PUBLICADOS }\end{array}$ \\
\hline Di Serio, L. C. & 36 & $88,8 \%$ & 32 \\
\hline Moori, R. G. & 31 & $90,2 \%$ & 32 \\
\hline Pereira, S. C. F. & 25 & $84,6 \%$ & 40 \\
\hline Csillag, J. M. & 25 & $85,5 \%$ & 18 \\
\hline Erdmann, R. H. & 24 & $85,3 \%$ & 21 \\
\hline Paiva, E. L. & 22 & $85,3 \%$ & 13 \\
\hline Carpinetti, L. C. R. & 21 & $82,6 \%$ & 16 \\
\hline Martins, R. S. & 21 & $82,6 \%$ & 17 \\
\hline Morabito, R. & 20 & $88,7 \%$ & 23 \\
\hline Pires, S. R. I. & 20 & $84,1 \%$ & \\
\hline
\end{tabular}


Os resultados das hipóteses $2_{\mathrm{a}}, 3_{\mathrm{a}}$ e $4_{\mathrm{a}}$, que envolvem o número de artigos publicados como variável dependente, estão na Tabela 6. No Modelo 1, que envolve a amostra como um todo, pode-se observar o efeito da colaboração (degree) e da eficiência dos laços por meio das lacunas estruturais sobre a autoria. A variável degree está positiva e significativamente relacionada com o número de artigos publicados, corroborando a hipótese $2_{\mathrm{a}}$. Na média, para cada novo colaborador, um pesquisador publica adicionalmente 0,78 artigo. Acerca da eficiência dos laços (efficiency), também houve influência positiva e significativa sobre a autoria, corroborando a hipótese 3 a. Para um aumento de $10 \%$ no percentual de laços não redundantes, há um incremento de 0,35 artigo.

O modelo aponta que a efetiva capacidade de um pesquisador em explorar as lacunas estruturais (structural holes), assim como de estabelecer maior quantidade de laços, contribui para o volume de artigos que ele publica no campo. Conforme apontam Lin (2001) e Mizruchi (1996), a exploração dessas lacunas implica receber informação, recursos e conhecimento por meio dos seus contatos e pressupõe que, quanto maior a diversidade dos laços de um indivíduo na rede, maior o acesso a recursos diferentes do grupo social que interage com maior frequência.

Os modelos 2 e 3 envolvem a divisão da amostra em dois grupos, objetivando testar o efeito moderador do degree sobre a relação ente eficiência dos laços e número de autorias $\left(\mathrm{H}_{4 \mathrm{a}}\right)$. No Modelo 2 , está exposto o efeito das lacunas estruturais considerando somente os 164 pesquisadores com maior número de laços. Nesse grupo, para um aumento de $10 \%$ na eficiência das lacunas estruturais há um aumento de 3,64 artigos publicados por autor, sendo tal relação significativa. Já o Modelo 3, que envolve o efeito das lacunas estruturais sobre a produção, tem como referência os 2.984 pesquisadores com menor número de colaboradores. Nesse grupo, para um aumento em 10\% na eficiência das lacunas estruturais, há um aumento de 0,12 artigo publicado, sendo tal relação também significativa. Como destacado pelo coeficiente de explicação dos dois modelos, enquanto no grupo de pesquisadores com maior colaboração as lacunas estruturais explicam 38\% da autoria, no grupo de autores com menor colaboração o coeficiente de explicação é de somente 3\%, evidenciando que a relação entre as variáveis é mais forte no grupo de maior colaboração. Comparando o valor de beta entre os dois modelos, verifica-se que a diferença entre os coeficientes das lacunas estruturais nos dois grupos é significativa, ou seja, há influência maior dessa variável entre aqueles que têm maior número de colaboradores do que os demais, corroborando a hipótese $4_{a}$. Essa relação pode ser visualmente verificada por meio da Figura 2. 
FÓRUM - GESTÃO DE OPERAÇÕES NO BRASIL: UMA ANÁLISE DO CAMPO CIENTÍFICO A PARTIR DA REDE SOCIAL DE PESQUISADORES

Guilherme Silveira Martins - Luciano Rossoni - João Mário Csillag - Michele Esteves Martins - Susana Carla Farias Pereira

Tabela 6 - Efeito da centralidade e da eficiência dos laços sobre a autoria (1997-2009)

\begin{tabular}{|l|c|c|c|c|}
\hline \multirow{2}{*}{ Degree } & TOTAL & $\begin{array}{c}\text { ALTO } \\
\text { DEGREE }\end{array}$ & $\begin{array}{c}\text { BAIXO } \\
\text { DEGREE }\end{array}$ & MODERAÇÃO \\
\cline { 2 - 5 } & MODELO 1 & MODELO 2 & MODELO 3 & MODELO DE ARNOLD (1982) \\
\hline Efficiency (\% structural holes) & $0,779 * * *$ & & & \\
\hline & $(0,008)$ & & & \\
\hline Constante & $3,490 * * *$ & $36,347 * * *$ & $1,183 * * *$ & $35,163 * * *$ \\
\hline & $(0,122)$ & $(3,670)$ & $(0,122)$ & {$[91,681]$} \\
\hline $\mathrm{N}$ & $-1,597 * * *$ & $-16,231 * * *$ & $1,014 * * *$ & - \\
\hline $\mathrm{F}$ & $(0,061)$ & $(2,598)$ & $(0,057)$ & - \\
\hline $\mathrm{R}{ }^{2}$ & 3148 & 164 & 2984 & - \\
\hline $\mathrm{R}{ }^{2}$ ajustado & 4619,05 & 98,069 & 92,762 & - \\
\hline $\mathrm{p}$-valor & 74,6 & 0,38 & 0,03 & - \\
\hline
\end{tabular}

Notas: Erro padrão entre parênteses. Moderação = (beta modelo 2 - beta modelo 3 ). Resultado do teste do qui quadrado de comparação dos betas entre colchetes. P-valor: *** $\mathrm{p}<0,01 ; * * \mathrm{p}<0,05 ; * \mathrm{p}<0,1$.

Por fim, os resultados que corroboram ou não as hipóteses $2_{b}, 3_{b}$ e 4 b, que envolvem como variável dependente a produtividade, estão na Tabela 7. No Modelo 1, pode-se observar que o efeito da colaboração (degree) sobre a produtividade é negativo, levando-se a rejeitar a hipótese 2 b. Apesar de a influência ser pequena, tais resultados vão ao encontro do que fora anteriormente esboçado por Rossoni e Machado-da-Silva (2008), que o aumento na prática de cooperação tem afetado a produtividade média dos pesquisadores. Nesse mesmo modelo é possível verificar a influência das lacunas estruturais sobre a produtividade, em que essa se mostrou positiva e significativa, corroborando a hipótese $3_{\mathrm{b}}$. Para um aumento de $10 \%$ no percentual de laços não redundantes, há um incremento de 10,5\% na produtividade dos pesquisadores.

Figura 2 - Efeito moderador da centralidade de grau

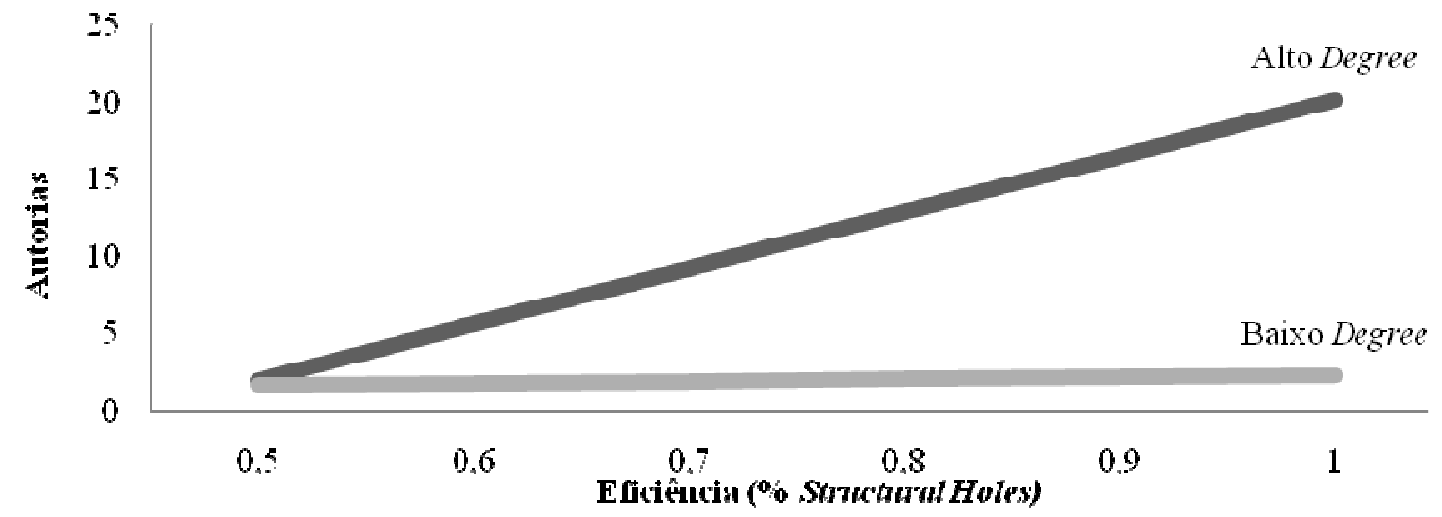


Em relação à moderação, podemos observar que, nos modelos 2 e 3, a influência das lacunas estruturais sobre a produtividade também foi significativa, no entanto a diferença entre os coeficientes beta foi bem pequena, não sendo tal diferença significativa, como apontado pelo teste de diferenças de coeficientes entre os dois grupos. Sendo assim, rejeita-se a hipótese $4_{b}$, não havendo moderação da centralidade de grau (degree) na relação entre eficiência dos laços e produtividade.

Tabela 7 - Efeito da centralidade e da eficiência dos laços sobre a produtividade (1997-2009)

\begin{tabular}{|l|c|c|c|c|}
\hline \multirow{2}{*}{} & TOTAL & $\begin{array}{c}\text { ALTO } \\
\text { DEGREE }\end{array}$ & $\begin{array}{c}\text { BAIXO } \\
\text { DEGREE }\end{array}$ & MODERAÇÃO \\
\cline { 2 - 5 } & MODELO 1 & MODELO 2 & MODELO 2 & MODELO DE ARNOLD (1982) \\
\hline Degree & $-0,004^{* *}$ & & & \\
\hline Efficiency (\% structural holes) & $(0,001)$ & & & 0,023 \\
\hline & $1,054^{* *}$ & $1,084^{* * *}$ & $1,060^{* * *}$ & {$[0,009]$} \\
\hline Constante & $(0,026)$ & $(0,243)$ & $(0,028)$ & - \\
\hline & $0,078^{* * *}$ & 0,019 & $0,066^{* * *}$ & - \\
\hline $\mathrm{N}$ & $(0,013)$ & $(0,172)$ & $(0,013)$ & - \\
\hline $\mathrm{F}$ & 3148 & 164 & 2984 & - \\
\hline $\mathrm{R}^{2}$ & 774,87 & 19,802 & 1407,494 & - \\
\hline $\mathrm{R}{ }^{2}$ ajustado & 0,33 & 0,11 & 0,32 & - \\
\hline p-valor & 0,33 & 0,10 & 0,32 & - \\
\hline Fonte: & $<0,001$ & $<0,001$ & $<0,001$ & \\
\hline
\end{tabular}

Fonte: resultados da pesquisa. Notas: Erro padrão entre parênteses. Moderação $=$ (beta modelo $2-$ beta modelo 3 ). Resultado do teste de qui quadrado de comparação dos betas entre colchetes. P-valor: *** $\mathrm{p}<0,01 ; * * \mathrm{p}<0,05 ; * \mathrm{p}<0,1$.

\section{CONCLUSÕES E RECOMENDAÇÕES}

Este trabalho partiu do pressuposto de que o conhecimento científico é construído socialmente (MERTON, 1973; KUHN, 1978), e buscou analisar a rede de pesquisadores do campo de Gestão de Operações no Brasil. Ao longo do período analisado, observou-se um crescimento exponencial do campo, com maior número de pesquisadores que publicam na área, sendo que a confecção dos artigos tem sido efetuada com uma estrutura de colaboração mais diversificada. Tal crescimento do número de publicações, de pesquisadores e da cooperação também foi observado em outros campos da Administração no Brasil (ROSSONI e GUARIDO-FILHO, 2007; ROSSONI e MACHADO-DASILVA, 2008) e foi influenciado pelos critérios da Coordenação de Aperfeiçoamento de Pessoal de 
Nível Superior (Capes), órgão responsável no país pela avaliação dos programas de pós-graduação stricto sensu.

As hipóteses testadas neste trabalho visam contribuir para um melhor entendimento da Gestão de Operações no Brasil enquanto campo científico, bem como da dinâmica dos pesquisadores que nele atuam. Inicialmente, a configuração da rede como small world aponta que os pesquisadores formam grupos coesos de colaboração, mas, ao mesmo tempo, esses grupos não são isolados uns dos outros. Tal estrutura da rede é benéfica para que os pesquisadores potencializem a troca de informações, de modo a fomentar o compartilhamento de significados ou o intercâmbio de conceitos e técnicas de pesquisa.

Outro aspecto relevante desse achado é o fato de uma rede do tipo small world ser menos suscetível à fragmentação (KOGUT e WALKER, 2001). Isso possibilita uma maior estabilidade dos relacionamentos sem afetar a capacidade criativa dos indivíduos na rede. Com tais condições, tem-se maior possibilidade de permanência das formas de conduta relativas à prática de pesquisa científica, especialmente em momentos de expansão, como aqueles pelos quais vem passando a academia brasileira em Administração. Com isso, não se pretende afirmar que há rigidez na estrutura da rede, o que poderia suscitar questionamentos acerca do determinismo dos relacionamentos sociais sobre a prática da pesquisa. Todavia, é possível observar maior estabilidade na natureza dos relacionamentos a partir da queda do ritmo de crescimento dos indicadores estruturais da rede.

O posicionamento dos pesquisadores na rede também revelou aspectos relevantes sobre a estrutura dos relacionamentos e como ela se relaciona com a produção de artigos no campo. Os resultados evidenciam empiricamente que tanto a quantidade de laços quanto a sua diversidade (contatos em diferentes grupos) possui relação positiva e significante com o volume de artigos publicados pelo pesquisador e com sua produtividade. Adicionalmente, verificou-se que o efeito dos laços não redundantes é estratificado pela quantidade de laços, em que pesquisadores com maior número de colaboradores exploram melhor os benefícios de tais laços do que os demais. No que tange à produção de artigos, parece ser mais contributivo estabelecer relacionamento com pesquisadores que não são contatos dos seus atuais coautores, aumentando a diversidade das relações. Em coerência com teorias sobre o capital social dos laços fracos (BURT, 1992, 2000; GRANOVETTER, 1983; LIN, 2001), esse esforço permitiria explorar novos recursos a partir do acesso a grupos sociais diversos.

A elaboração deste estudo abre perspectivas para que pesquisas futuras sobre este tema sejam realizadas. Parece interessante examinar, a partir de uma abordagem qualitativa, os antecedentes e as estratégias dos pesquisadores na construção da sua rede de colaboração. Outros estudos podem explorar 
as limitações deste trabalho. Uma delas está vinculada ao tipo de relacionamento escolhido para análise, dado que os pesquisadores não cooperam apenas pelo meio formal de publicação de artigos científicos. Existem relações entre orientadores e orientandos, de participação em bancas de mestrado e doutorado, coordenação de programas, dentre outras. Outra limitação está relacionada à delimitação da pesquisa. Os periódicos e os eventos estudados representam uma parcela dos locais em que os artigos científicos de Gestão de Operações são publicados. Contudo, esses são os principais fóruns da área, o que torna o recorte representativo.

\section{REFERÊNCIAS}

ACEDO, F. J. e outros. Co-authorship in management and organizational studies: an empirical and network analysis. Journal of Management Studies, v. 43, n. 5, p. 957-983, 2006.

AMOAKO-GYAMPAH, K; MEREDITH, J. R. The operations management research agenda: an update. Journal of Operations Management, v. 8, n. 3, p. 250-262, 1989.

ARKADER, R. A pesquisa científica em gerência de operações no Brasil. RAE-revista de administração de empresas, v. 43, n.1, p. 70-80, 2003.

ARNOLD, H. J. Moderator variables: a clarification of conceptual, analytic, and psychometric issues. Organizational Behavior and Human Performance, v. 29, n. 2, p. 143-174, 1982.

BARABÁSI, A; ALBERT, R. Emergence of scaling in random networks. Science, v. 286, n. 5439, p. 509-512, 1999.

BORGATTI, S. Structural holes: unpacking Burt's redundancy measures. Connections, v. 20, n. 1, p. 35-38, 1997.

BOYER, K; SWINK, M; ROSENZWEIG, E. Operations strategy research in the POMS Journal. Production and Operations Management, v. 14, n. 4, p. 442, 2005. 
BRAUN, T; GLÄNZEL, W; SCHUBERT, A. Publication and cooperation patterns of the authors of neuroscience journals. Scientometrics, v. 51, n. 3, p. 499-510, 2001.

BUFFA, E.S. Research in operations management. Journal of Operations Management, v. 1, n. 1, p. 18,1980 .

BULGACOV, S; VERDU, F. Redes de pesquisadores da área de administração: um estudo exploratório. RAC - Revista de Administração Contemporânea, Edição Especial, p. 163-182, 2001.

BURT, R. The structure of social capital. In: SUTTON, R; STAW, B. M. Research in Organizational Behavior, v. 22. Greenwich: JAI Press, 2000. p. 345-423.

BURT, R. Structural Holes: The Social Structure of Competition. Cambridge: Harvard University Press, 1992.

GLANZEL, W; SCHUBERT, A. Analyzing scientific network through co-authorship. Handbook of Quantitative Science and Technology Research, 2004.

GRANOVETTER, M. The Strength of Weak Ties. American Journal of Sociology, v. 78, n. 6, p. 13611380, 1973.

GUPTA, S; VERMA, R; VICTORINO, L. Empirical research published in production and operations management (1992-2005): trends and future research directions. Production and Operations Management, v. 15, n. 3, p. 432-448, 2006.

KOGUT, B, WALKER, G. The small world of Germany and the durability of national networks. American Sociological Review, v. 66, n. 3, 317-335, 2001.

KUHN, T. A estrutura das revoluções científicas. 2. ed. São Paulo: Perspectiva, 1978. 
FÓRUM - GESTÃO DE OPERAÇÕES NO BRASIL: UMA ANÁLISE DO CAMPO CIENTÍFICO A PARTIR DA REDE SOCIAL DE PESQUISADORES

Guilherme Silveira Martins - Luciano Rossoni - João Mário Csillag - Michele Esteves Martins - Susana Carla Farias Pereira

LEE, S; BOZEMAN, B. The impact of research collaboration on scientific productivity. Social Studies of Science, v. 35, n. 5, p. 673-702, 2005.

LIN, N. Social Capital: A Theory of Social Structure and Action. Cambridge: Cambridge University Press, 2001.

LOTKA, A. J. The frequency distribution of scientific productivity. Journal of the Washington Academy of Sciences, v. 16, n. 12, 317-323, 1924.

MERTON, R. The Matthew effect in science. Science, v. 159, n. 3810, p. 56-63, 1968.

MERTON, R. The Sociology of Science. Chicago: University of Chicago Press, 1973.

MERTON, R. The Matthew effect in science, II: cumulative advantage and the symbolism of intellectual property. Isis, v. 79, n. 4, p. 606-623, 1988.

MERTON, R. On social structure and science. Chicago: Chicago University Press, 1996.

MIZRUCHI, M. What do interlocks do? An analysis, critique, and assessment of research on interlocking directorates. Annual Review of Sociology, v. 22, p. 271-298, 1996.

MOODY, J. The structure of a social science collaboration network: disciplinary cohesion from 1963 to 1999. American Sociological Review, v. 69, p. 213-238, 2004.

NEWMAN, M. Coauthorship networks and patterns of scientific collaboration. Proceedings of the National of Academic Sciences, v. 101, p. 5200-5205, 2004.

NEWMAN, M. The structure of scientific collaboration networks. Proceedings of National Academic Sciences, v. 98, n. 2, 404-409, 2001. 
FÓRUM - GESTÃO DE OPERAÇÕES NO BRASIL: UMA ANÁLISE DO CAMPO CIENTÍFICO A PARTIR DA REDE SOCIAL DE PESQUISADORES

Guilherme Silveira Martins - Luciano Rossoni - João Mário Csillag - Michele Esteves Martins - Susana Carla Farias Pereira

PANNIRSELVAM, G. e outros. Operations management research: an update for the 1990s. Journal of Operations Management, v. 18, p. 95-112, 1999.

PRASAD, S; BABBAR, S. Operations management research: an update for the 1990s. Journal of Operations Management, v.18, p.209-247, 2000.

PREACHER, K. J; RUCKER, D. D; HAYES, A. F. Addressing moderated mediation hypotheses: theory, methods, and prescriptions. Multivariate Behavioral Research, v. 42, n. 1, p. 185-227, 2007.

RODRIGUES, S; CARRIERI, A. A Tradição anglo-saxônica nos estudos organizacionais brasileiros. RAC - Revista de Administração Contemporânea, Edição Especial, p. 81-102, 2001.

ROSSONI, L; GUARIDO-FILHO, E. Cooperação interinstitucional no campo da pesquisa em estratégia. Revista de Administração de Empresas, v. 47, n. 4, p. 74-87, 2007.

ROSSONI, L; GUARIDO-FILHO, E. Cooperação entre programas de pós-graduação em administração no Brasil: evidências estruturais em quatro áreas temáticas. Revista de Administração Contemporânea, v. 13, n. 3, p. 366-390, 2009.

ROSSONI, L; HOCAYEN-DA-SILVA, A. Cooperação entre pesquisadores da área de administração da informação: evidências estruturais de fragmentação das relações no campo científico. Revista de Administração da USP, v. 43, n. 2, p. 138-151, 2008.

ROSSONI. L, MACHADO-DA-SILVA, C. L. Análise institucional da construção do conhecimento científico em mundos pequenos. Faces, v. 7, n. 1, 25-43, 2008.

SCOTT, J. Social Network Analysis: A Handbook. 2. ed. London: Sage Publications, 2000.

SOTERIOU, A. e outros. Assessing production and operations management related journals: the European perspective. Journal of Operations Management, v.17, p.225-238, 1999. 
FÓRUM - GESTÃO DE OPERAÇÕES NO BRASIL: UMA ANÁLISE DO CAMPO CIENTÍFICO A PARTIR DA REDE SOCIAL DE PESQUISADORES

Guilherme Silveira Martins - Luciano Rossoni - João Mário Csillag - Michele Esteves Martins - Susana Carla Farias Pereira

SPRAGUE, L. Evolution of the field of operations management. Journal of Operations Management, v. 25, p. 219-238, 2007.

SWAMIDASS, P. Empirical science: new frontier in operations management research: a citation analysis. Journal of Operations Management, v. 14, p. 345-355, 1996.

UZZI, B; SPIRO, J. Collaboration and creativity: the small world problem. American Journal of Sociology, v. 111, n. 2, p. 447-504, 2005.

WAGNER, C; LEYDESDORFF, L. Network structure, self-organization, and the growth of international collaboration in science. Research Policy, v. 34, n. 10, p. 1608-1618, 2005.

WASSERMAN, S; FAUST, K. Social Network Analysis: Methods and Applications. Cambridge: Cambridge University Press, 1994.

WATTS, D. Small Worlds: The Dynamics of Networks Between Order and Randomness. Princeton: Princeton University Press, 1999.

WATTS, D; STROGATZ, S. Collective dynamics of "small-world" networks. Nature, v. 393, p. 440442, June 1998. 